\title{
Outcomes of patients treated with Sengstaken- Blakemore tube for uncontrolled variceal hemorrhage
}

\author{
Ja Yun Choi ${ }^{1,}$, , Yun Won Jo ${ }^{1,}$, , Sang Soo Lee ${ }^{1,2}$, Wan Soo Kim ${ }^{1,2}$, Hye Won Oh ${ }^{1}$, Cha Young Kim ${ }^{1}$, \\ Eun Young Yun ${ }^{1}$, Jin Joo Kim, ${ }^{1,2}$ Jae Min Lee ${ }^{1,2}$, Hong Jun $\mathrm{Kim}^{1}$, Hyun Jin Kim, ${ }^{1,2,3}$, Tae Hyo Kim ${ }^{1,3}$, \\ Woon Tae Jung ${ }^{1,3}$, Ok Jae Lee ${ }^{1,3}$, and Rock Bum Kim ${ }^{4}$
}

\begin{abstract}
${ }^{1}$ Department of Internal Medicine, Gyeongsang National University Hospital, Jinju; ${ }^{2}$ Department of Internal Medicine, Gyeongsang National University Changwon Hospital, Changwon; ${ }^{3}$ Institute of Health Sciences, Gyeongsang National University, Jinju; ${ }^{4}$ Regional Cardiocerebrovascular Disease Center, Gyeongsang National University School of Medicine, Jinju, Korea
\end{abstract}

Received: October 25, 2016 Revised : December 1, 2016 Accepted: February 8, 2017

\section{Correspondence to Sang Soo Lee, M.D. \\ Department of Internal Medicine, Gyeongsang National University Changwon Hospital, 11 Samjeongja-ro, Seongsan-gu, Changwon 51472, Korea \\ Tel: +82-55-214-3711 \\ Fax: $+82-55-214-1036$ \\ E-mail:3939lee@naver.com}

*These authors contributed equally to this work.
Background/Aims: The aim of this study was to investigate the mortality, bleeding control rate, and their associated predictors in patients treated with Sengstaken-Blakemore (SB) tube for uncontrolled variceal hemorrhage associated with hemodynamic instability or failure of endoscopic treatment.

Methods: The clinical data of 66 consecutive patients with uncontrolled variceal hemorrhage treated with SB tube at Gyeongsang National University Hospital from October 2010 to October 2015 were retrospectively analyzed.

Results: The overall success rate of initial hemostasis with SB tube was $75.8 \%$, and the independent factors associated with hemostasis were non-intubated state before SB tube (odds ratio, 8.50; $p=0.007$ ) and Child-Pugh score $<11$ (odds ratio, 15.65; $p=0.022$ ). Rebleeding rate after successful initial hemostasis with SB tube was $22.0 \%$, and esophageal rupture occurred in $6.1 \%$. Mortality within 30 days was $42.4 \%$, and the related independent factors with mortality were failure of initial hemostasis with SB tube (hazard ratio, 6.24; $p<0.001$ ) and endotracheal intubation before SB tube (hazard ratio, $2.81 ; p=0.018$ ).

Conclusions: Since the era of endoscopic band ligation, SB tube might be a beneficial option as a temporary salvage treatment for uncontrolled variceal hemorrhage. However, rescue therapy had a high incidence of fatal complication and rebleeding.

Keywords: Variceal hemorrhage; Sengstaken-Blakemore tube; Mortality; Hemostasis

\section{INTRODUCTION}

Variceal hemorrhage is a life-threatening emergency and a major cause of death in patients with liver cirrhosis. The mortality of acute variceal hemorrhage is reportedly $20 \%$ at 6 weeks $[1,2]$. Until 1980, the main management of acute variceal hemorrhage was based on balloon tamponade [3]. Current recommendation for acute variceal hemorrhage in patients with liver cirrhosis is combined management with vasoactive drugs, endoscopic therapy, and prophylactic antibiotics [1]. This change has resulted in a great improvement in mortality from $42.6 \%$ in 1980 to $14.5 \%$ in 2000 since the era of the endoscopic band ligation [4]. Prior to the 1970s, surgical shunting was used as a rescue therapy for uncontrolled variceal hemorrhage, but was superseded by the trans- 
jugular intrahepatic portosystemic shunt (TIPS) in the late 1990s [5]. Recent guideline still suggests a role for balloon tamponade; however, it should only be used as a temporary measure in massive variceal hemorrhage until definitive treatment when endoscopic therapy fails or is not possible [1].

The Sengstaken-Blakemore (SB) tube was originally described in 1950 by Sengstaken and Blakemore [6]. The effectiveness of SB tube for hemostasis in initial control of variceal hemorrhage was reportedly 50\% to $90 \%$ until 1980 [7-11]. However, since the era of the endoscopic band ligation, bleeding control rate for variceal hemorrhage was $80 \%$ to $97 \%$ [12], but there are no reports on outcomes of SB tube in rescue therapy for uncontrolled variceal hemorrhage associated with hemodynamic instability or failure of endoscopic treatment. The aim of this study was to investigate mortality, overall success rate of initial hemostasis, and their associated predictors in patients treated with SB tube for uncontrolled variceal hemorrhage associated with hemodynamic instability or failure of endoscopic treatment.

\section{METHODS}

\section{Study population}

The subjects were 512 patients consecutively diagnosed with liver cirrhosis and received therapeutic endoscopy such as endoscopic band ligation and endoscopic injection of N-butyl-2-cyanoacrylate for treatment of variceal hemorrhage at Gyeongsang National University Hospital from October 2010 to October 2015. During the study period, 66 patients diagnosed with uncontrolled variceal hemorrhage were treated with SB tube. All patients who met the following criteria were included: (1) diagnosis with liver cirrhosis based on the presence of portal hypertension and compatible imaging findings; (2) variceal hemorrhage was confirmed on endoscopy; (3) uncontrolled variceal hemorrhage associated with hemodynamic instability or failure of endoscopic treatment; and (4) rescue therapy by SB tube. In 33 cases, emergency endoscopy before SB tube insertion was performed to achieve hemostasis. In 33 patients, SB tube was applied without emergency endoscopy under the suspicion of variceal hemorrhage: only following the endoscopy was performed after SB tube insertion at same admission be- cause patients could not undergo endoscopy before SB tube due to hemodynamic instability or severe encephalopathy (Fig. 1). SB tube was maintained and removed until no bleeding occurred for 24 hours or until the next endoscopic therapy. The Institutional Review Board of Gyeongsang National University Hospital reviewed and approved the study (IRB file No. 2015-01-008-004).

\section{Data collection and treatment methods}

Demographic, etiology of cirrhosis, biochemical laboratory, Child-Pugh score, the model for end-stage liver disease (MELD) score, grade of ascites and encephalopathy, previous variceal hemorrhage, initial vital sign at hemorrhage, endoscopic finding, endotracheal intubation before SB tube insertion, time of hemorrhage and SB tube insertion, reasons of SB tube insertion, and cause of death were retrieved from electronic medical records.

In the current study, prophylactic antibiotics and

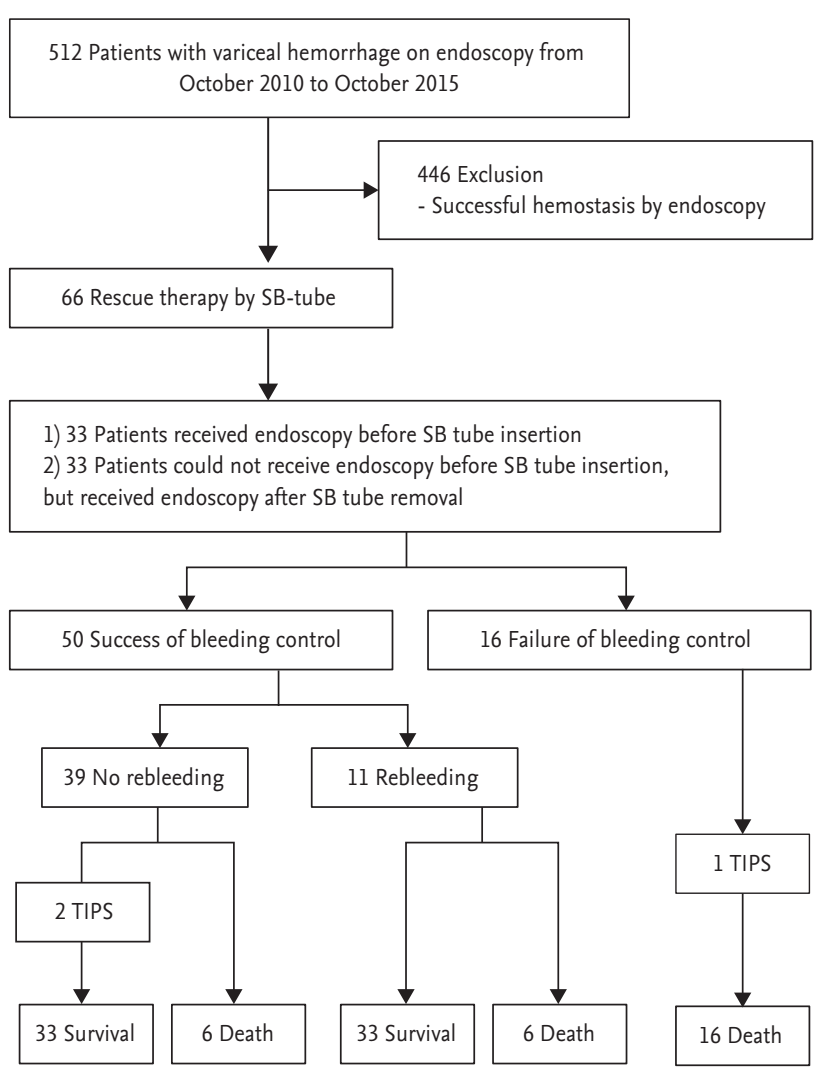

Figure 1. Outcomes of patients treated with Sengstaken-Blakemore (SB) tube for uncontrolled variceal hemorrhage. Emergency transjugular intrahepatic portosystemic shunt (TIPS) was not available in our center. 
vasoconstrictors such as terlipressin or somatostatin were administered as soon as possible after variceal hemorrhage. Packed red blood cell was given to maintain hemoglobin of 7 to $8 \mathrm{~g} / \mathrm{dL}$ during hospitalization. Therapeutic endoscopy was performed within 24 hours after bleeding control unless patients could not undergo endoscopy due to hemodynamic instability or severe encephalopathy. If torrential bleeding with or without encephalopathy occurred, primary balloon tamponade therapy was used before endoscopy. If variceal bleeding was not controlled despite immediate endoscopic therapy, balloon tamponade or TIPS was used as a secondary temporary bridge therapy. Endoscopic therapy or TIPS was considered in rebleeding patients after primary control according to the attending physician's decision. However, the role of TIPS in active variceal hemorrhage in our center was exceedingly limited because emergency TIPS was not available.

\section{Outcome measures}

Successful initial hemostasis with SB tube was defined as cessation of bleeding more than 24 hours or until the next successful endoscopic therapy. Rebleeding was defined as the recurrence of bleeding after successful hemostasis during the same hospitalization. Ascites was classified into grades of 1 to 3 based on the definitions of the International Ascites Club [13]. The cause of death in this study could be divided into four categories: (1) failure of successful hemostasis, (2) rebleeding, (3) esophageal perforation, and (4) multi-organ failure.

\section{Statistical analysis}

Categorical variables were compared with the chi-square test or two-tailed Fisher exact test, and continuous variables were compared by the Mann-Whitney test. Univariate and multivariate Cox regression analyses were used to determine the correlation of the predictive factors for mortality and rebleeding. Logistic regression analyses were used to determine the correlation of the predictive factors for bleeding control. Variables that were significant in the univariate analysis $(p<0.05)$ were included in a multivariate analysis using Cox and logistic regression model. Risk analysis was expressed by the odds ratio (OR) or hazard ratio (HR) and the $95 \%$ confidence interval (CI). A two-tailed $p<0.05$ was considered as statistically significant. Statistical analyses were performed using PASW version 18.0 (SPSS Inc., Chicago, IL, USA).

\section{Ethics approval}

All procedures performed in studies involving human participants were in accordance with the ethical standards of the Institutional Review Board of the Gyeongsang National University Hospital and with the 1964 Helsinki declaration and its later amendments or comparable ethical standards. Informed consent was not applicable (due to the retrospective study).

\section{RESULTS}

\section{Baseline characteristics}

We identified a total of 66 cirrhotic patients with uncontrolled variceal hemorrhage who were treated with $\mathrm{SB}$ tube (Table 1). Most subjects were male ( $\mathrm{n}=62$, 93.9\%) and the median age was 53 years. The etiologies of cirrhosis were alcohol in 49 patients (74.2\%), combination of virus (hepatitis B or C infection) and alcohol in eight patients (12.1\%), and virus in nine patients (13.6\%). Fourteen patients (21.2\%) had hepatocellular carcinoma, and 47 patients $(71.2 \%)$ had a history of previous variceal hemorrhage treated with endoscopic variceal ligation or N-butyl cyanoacrylate injection. The median MELD score and Child-Pugh score at the time of initial hemorrhage was 18.5 and 11.o. There was no significant difference in the number of hemorrhages or number of SB tube insertions between the daytime and nighttime (data not shown).

\section{Management of variceal hemorrhage}

The management of variceal hemorrhage at the time of initial hemorrhage was shown in Table 2. The sources hemorrhage were esophageal varices in 55 (83.3\%) and gastric varices in 11 (16.7\%). At the time of initial hemorrhage, all patients received prophylactic intravenous antibiotics, in addition to terlipressin. Among the 66 patients treated with SB tube, 33 (50.0\%) received endoscopy at the time of initial hemorrhage before SB tube insertion and 18 (27.3\%) underwent endotracheal intubation before SB tube insertion. Variceal hemorrhage was controlled with the gastric balloon in $16(24.2 \%)$ and with both gastric and esophageal balloons in 50 patients (75.8\%). Reasons of SB tube insertion were severe en- 
Table 1. Baseline characteristics of patients who were treated with Sengstaken-Blakemore tube for acute uncontrolled variceal hemorrhage

\begin{tabular}{lc}
\hline Characteristic & Value $(\mathrm{n}=66)$ \\
\hline Male sex & $62(93.9)$ \\
Age, yr & $53.0(46.8-59.5)$ \\
Etiology of cirrhosis, alcohol/viral & $49 / 8 / 9(74.2 / 12.1 / 13.6)$ \\
+ alcohol/viral & \\
Hepatocellular carcinoma & $14(21.2)$ \\
Previous variceal hemorrhage & $47(71.2)$ \\
Ascites, absent/mild to moderate/ & $18 / 26 / 22(27.3 / 39.4 / 33.3)$ \\
severe & \\
Overt encephalopathy & $28(42.4)$ \\
Child-Pugh score & $11.0(8.0-12.3)$ \\
MELD score & $18.5(13.0-27.3)$ \\
Hemoglobin, g/dL & $7.5(6.2-9.2)$ \\
Platelet, $\times 1,0 \circ 0 / \mu \mathrm{L}$ & $90.0(66.5-120.8)$ \\
Bilirubin, g/dL & $2.5(1.5-4.5)$ \\
INR & $1.83(1.49-2.75)$ \\
Albumin, g/dL & $2.6(2.0-2.9)$ \\
AST, IU/L & $79.0(50.5-126.8)$ \\
ALT, IU/L & $27.5(19.8-56.3)$ \\
Na, mmol/L & $136.8(133.5-139.2)$ \\
WBC, 10 $\times 109 / \mathrm{L}$ & $8.7(6.2-13.0)$ \\
\hline
\end{tabular}

Values are presented as number (\%) or median (interquartile range).

MELD, model for end-stage liver disease; INR, international normalized ratio; AST, aspartate aminotransferase; ALT, alanine aminotransaminase; WBC, white blood cell. cephalopathy in six patients (9.1\%), failure of endoscopic hemostasis in 26 patients (39.4\%), and hemodynamic instability in 34 patients (51.5\%).

\section{Outcomes of patients}

The outcomes of these patients were shown in Table 3. SB tube successfully controlled hemorrhage in 50 patients $(75.8 \%)$ in whom endoscopic therapy had failed or was not possible. Among 50 patients, 11 (22.0\%) had rebleeding after successful hemostasis with SB tube. Perforation of esophagus was encountered in four pa-

Table 2. Management of variceal hemorrhage at the time of initial hemorrhage in patients with SB tube $(n=66)$

\begin{tabular}{|c|c|}
\hline Characteristic & No. (\%) \\
\hline $\begin{array}{l}\text { Source of hemorrhage, esophageal/ } \\
\text { gastric varix }\end{array}$ & $55 / 11(83.3 / 16.7)$ \\
\hline Endoscopy before SB tube insertion & $33(50.0)$ \\
\hline $\begin{array}{l}\text { Endotracheal intubated state before SB } \\
\text { tube insertion }\end{array}$ & $18(27 \cdot 3)$ \\
\hline Terlipressin & $66(100)$ \\
\hline $\begin{array}{l}\text { Gastric balloon/gastric and esophageal } \\
\text { balloons }\end{array}$ & $16 / 50(24.2 / 75.8)$ \\
\hline \multicolumn{2}{|l|}{ Reasons of SB tube insertion } \\
\hline Severe encephalopathy & $6(9.1)$ \\
\hline Failure of endoscopic hemostasis & $26(39.4)$ \\
\hline Hemodynamic instability & $34(51.5)$ \\
\hline
\end{tabular}

SB, Sengstaken-Blakemore.

Table 3. Outcomes of patients who were treated with SB tube for acute uncontrolled variceal hemorrhage

\begin{tabular}{lc}
\hline Outcome & No. $(\%)$ \\
\hline Initial hemostasis with SB tube $(\mathrm{n}=66)$ & $50(75.8)$ \\
Rebleeding after successful hemostasis with SB tube $(\mathrm{n}=50)$ & $11(22.0)$ \\
Esophageal perforation $(\mathrm{n}=66)$ & $4(6.1)$ \\
Death $(\mathrm{n}=66)$ & $28(42.4)$ \\
Causes of death $(\mathrm{n}=28)$ & $12(42.9)$ \\
\hline After failed successful initial hemostasis with SB tube $(\mathrm{n}=16)$ & $4(14.3)$ \\
\hline Failure of successful hemostasis & \\
$\quad$ Esophageal perforation & \\
\hline After successful initial hemostasis with SB tube $(\mathrm{n}=12)$ & $5(17.9)$ \\
$\quad$ Among patients with rebleeding $(\mathrm{n}=6)$ & $1(3.6)$ \\
\hline Failed successful hemostasis & $6(21.4)$ \\
\hline Multi-organ failure & \\
\hline Among patients without rebleeding $(\mathrm{n}=6)$ & \\
\hline Multi-organ failure &
\end{tabular}

SB, Sengstaken-Blakemore. 
tients (6.1\%). Following an SB tube insertion, within 30 days the overall mortality rate was $42.4 \%$. Whereas, the 50 patients with a successful hemostasis outcome had an overall mortality rate of $24.0 \%$ (Fig. $2 \mathrm{~A}$ ). Cumulative survival rate in total 66 patients was significantly higher in patients with successful hemostasis than in those without successful hemostasis in log-rank test $(p<0.001)$ (Fig. 2A). Moreover, cumulative survival rate in total 66 patients was significantly higher in patients without endotracheal intubation than in those with endotracheal intubation $(p<0.001)$ (Fig. $2 B)$. Death was attributed to failure of bleeding control in 12 patients (42.9\%), rebleeding in five (17.9\%), esophageal perforation in four (14.3\%), and multi-organ failure in seven (25.0\%).

\section{Predictors of primary control and overall mortality}

SB tube controlled bleeding in 50 patients and was ineffective in 16 patients. Non-intubated state before SB tube (OR, 8.50; $p=0.007)$ and Child-Pugh score $<11(\mathrm{OR}$, 15.65; $p=0.022)$ were independent predictors related to
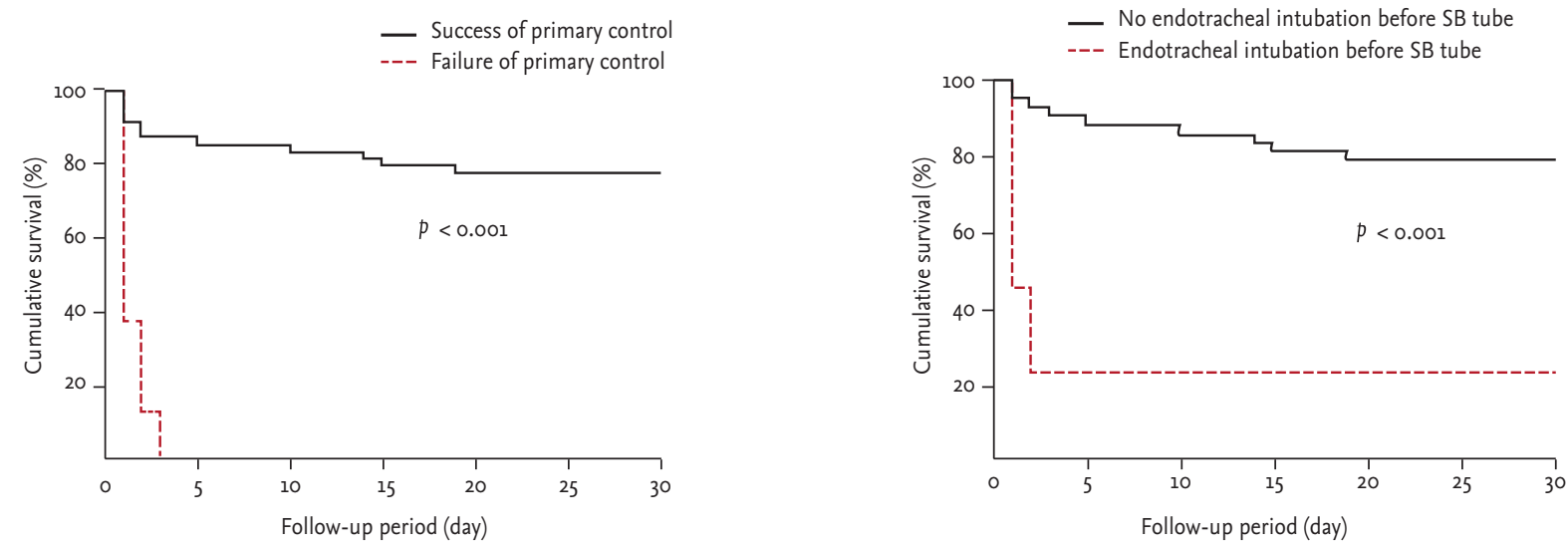

No. at risk for

$\begin{array}{lllllllll}\text { Success of primary control } & 50 & 42 & 41 & 39 & 38 & 38 & 38\end{array}$
Failure of primary control $\quad 16$

\section{A}

Figure 2. Cumulative survival rate according to success of initial hemostasis with Sengstaken-Blakemore tube (A) and endotracheal intubation (B).

Table 4. Independent predictors for initial hemostasis among patients who were treated with SB tube for acute variceal hemorrhage $(n=66)$

\begin{tabular}{|c|c|c|c|c|}
\hline \multirow{2}{*}{ Variable } & \multicolumn{2}{|c|}{ Univariate analysis } & \multicolumn{2}{|c|}{ Multivariate analysis } \\
\hline & OR $(95 \% \mathrm{CI})$ & $p$ value & OR $(95 \% \mathrm{CI})$ & $p$ value \\
\hline Age $<53$ yr & $1.67(0.53-5.29)$ & 0.386 & - & - \\
\hline Non-intubated state before SB tube & $8.75(2.48-30.95)$ & 0.001 & $8.50(1.80-40.25)$ & 0.007 \\
\hline Absence of overt HEP & $6.38(1.78-22.89)$ & 0.005 & - & - \\
\hline Hemoglobin $>8 \mathrm{~g} / \mathrm{dL}$ & $1.310(0.41-4.16)$ & 0.648 & - & - \\
\hline $\mathrm{WBC}<10 \times 10^{9} / \mathrm{L}$ & $5.13(1.52-17.35)$ & 0.008 & $3.89(0.65-23.20)$ & 0.136 \\
\hline INR $<1.83$ & $4.14(1.17-14.65)$ & 0.027 & - & - \\
\hline $\mathrm{Na}>135 \mathrm{mmol} / \mathrm{L}$ & $4.29(1.31-14.03)$ & 0.016 & $3.74(0.80-17.50)$ & 0.094 \\
\hline MELD $<19$ & $5.82(1.63-20.82)$ & 0.007 & $0.204(0.02-2.45)$ & 0.204 \\
\hline Child-Pugh score $<11$ & $10.50(2.15-51.28)$ & 0.004 & $15.65(1.35-181.48)$ & 0.022 \\
\hline
\end{tabular}

SB, Sengstaken-Blakemore; OR, odds ratio; CI, confidence interval; HEP, hepatic encephalopathy; WBC, white blood cell; INR, international normalized ratio; MELD, model for end-stage liver disease. 
Table 5. Independent predictors for 30 days mortality among patients treated with SB tube for acute variceal hemorrhage $(n=66)$

\begin{tabular}{|c|c|c|c|c|}
\hline \multirow{2}{*}{ Variable } & \multicolumn{2}{|c|}{ Univariate analysis } & \multicolumn{2}{|c|}{ Multivariate analysis } \\
\hline & OR $(95 \% \mathrm{CI})$ & $\mathrm{p}$ value & OR (95\% CI) & $p$ value \\
\hline Age $<53$ yr & $1.08(0.51-2.27)$ & 0.838 & - & - \\
\hline Ischemic hepatitis $^{\mathrm{a}}$ & $2.20(1.01-4.79)$ & 0.047 & $1.90(0.83-4.34)$ & 0.129 \\
\hline Endotracheal intubation before SB tube & $5.33(2.45-11.60)$ & $<0.001$ & $2.81(1.12-6.64)$ & 0.018 \\
\hline Failure of initial hemostasis with SB tube & $14.67(5.74-37.49)$ & $<0.001$ & $6.24(2.26-17.24)$ & $<0.001$ \\
\hline Overt HEP & $4.10(1.84-9.19)$ & 0.001 & - & - \\
\hline Moderate to severe ascites & $3.27(1.53-6.95)$ & 0.002 & - & - \\
\hline Hemoglobin $<8$ g/dL & $1.43(0.66-3.11)$ & 0.362 & - & - \\
\hline $\mathrm{WBC}<10 \times 10^{9} / \mathrm{L}$ & $3.49(1.60-7.62)$ & 0.002 & - & - \\
\hline INR $<1.83$ & $4.67(1.88-11.59)$ & 0.001 & - & - \\
\hline Albumin > 2.5 g/dL & $2.66(1.12-5.90)$ & 0.016 & - & - \\
\hline $\mathrm{Na}<135 \mathrm{mmol} / \mathrm{L}$ & $2.35(1.11-4.95)$ & 0.025 & $0.74(0.31-1.74)$ & 0.129 \\
\hline$M E L D \geq 18$ & $6.54(2.63-16.31)$ & $<0.001$ & $1.21(0.27-5.56)$ & 0.803 \\
\hline Child-Pugh score $\geq 11$ & $8.01(2.76-23.24)$ & $<0.001$ & $4.13(0.87-19.55)$ & 0.074 \\
\hline
\end{tabular}

SB, Sengstaken-Blakemore; HR, hazard ratio; CI, confidence interval; HEP, hepatic encephalopathy; WBC, white blood cell; INR, international normalized ratio; MELD, model for end-stage liver disease.

${ }^{a}$ Ischemic hepatitis: defined as aspartate aminotransferase $>500 \mathrm{IU} / \mathrm{L}$ or alanine aminotransaminase $>500$ IU/L within 24 hours.

initial hemostasis with SB tube in multivariate analysis (Table 4). The overall mortality at 30 days was $42.4 \%$. As shown in Table 5, failure of hemostasis with SB tube (HR, 6.24; $p<0.001)$ and endotracheal intubation (HR, 2.81; $p=0.018)$ were independent risk factors related to overall mortality in multivariate analysis. In addition, we also analyzed the predictors of rebleeding after successful initial hemostasis during the same hospitalization among 50 patients who achieved initial control. However, no factor was significantly related to rebleeding in univariate analysis (data not shown).

\section{DISCUSSION}

In the current study, the overall success rate of initial hemostasis by SB tube was $75.8 \%$ in acute uncontrolled variceal hemorrhage patients in whom endoscopic therapy had failed or was not possible, which was comparable to the reported rate before the era of endoscopic band ligation. Among 50 patients who achieved bleeding control with SB tube, 11 had rebleeding. Esophageal perforation was encountered in four patients. The overall mortality rate within 30 days was $42.4 \%$, but overall mortality rate in the 50 patients who achieved initial control was $24.0 \%$. Independent predictors related to initial hemostasis were non-intubated state before SB tube insertion and Child-Pugh score < 11. Moreover, independent risk factors related to overall mortality were failure of initial hemostasis with SB tube and endotracheal intubation before SB tube insertion.

In a previous report [4], the use of a balloon tamponade decreased steadily from $40 \%$ in 1980 to $6 \%$ in 2000 . A balloon tamponade was the first-line treatment for a variceal hemorrhage up until 1980; the primary control rate of balloon tamponade was reportedly 50\% to $90 \%$ [7-11,14,15]. The recommended current initial management for acute variceal hemorrhage is based on endoscopic therapy, where the rate of bleeding control is $80 \%$ to $97 \%$ [12]. However, in case of the treatment failing, rescue therapies, such as a balloon tamponade or TIPS may be necessary. In a prospective study conducted by Sorbi et al. [16] in 2003, a balloon tamponade was only used in $5.5 \%$ of patients who were experiencing variceal bleeding for the first time. In this study, all enrolled patients had uncontrolled severe bleeding and advanced liver cirrhosis with Child-Pugh class B or C. Therefore, we hypothesized that bleeding control rate by SB tube was lower than rates of previous reports (50\% to 90\%) $[7-11,14,15]$, because SB tube was only used in rescue ther- 
apy and in severe cases since the era of endoscopic band ligation. However, primary control rate of our result was comparable to that of previous reports. Besides, rebleeding rate (22.0\%) after SB tube insertion was comparable to that of previous reports (20\% to $50 \%)[10,11,17]$.

In previous studies the percentage of incidences due to SB tube complications, such as aspiration [7], asphyxia, or esophageal perforation was $10 \%$ to $45 \%$ [17]. In a study by Conn and Simpson [7], they found that major complications occurred in $35 \%$ of patients with an SB tube, while $22 \%$ of the patients did not survive. Esophageal perforation is a rare, but lethal complication. The rate of esophageal perforation due to SB tube is $2 \%$ to $10 \%[7,10,11,14,18]$. In our study, esophageal perforation occurred in four patients $(6.1 \%)$, in whom perforation due to misplaced SB tube was the main cause of death. Endotracheal intubation was followed by SB tube in all these patients. Among the 18 patients with endotracheal intubation before SB tube, four (22.2\%) developed esophageal perforation due to misplaced SB tube. Moreover, endotracheal intubation before SB tube insertion was an independent predictor for initial hemostasis with SB tube. Therefore, to prevent esophageal perforation, a careful assessment of the SB tube should be made before placement in cases with endotracheal intubation; in addition, confirmation of SB tube placement is recommended by X-ray and auscultation $[9,15,19]$.

Previous studies have reported a mortality rate of $20 \%$ to $30 \%$ for patients treated with a balloon tamponade [20-23]. In our study, the overall mortality rate was $42.4 \%$ at 30 days. This could suggest that since the era of the endoscopic band ligation, SB tube rescue therapies may have a higher mortality rate than if SB tubes were being used as the first-line of treatment for variceal hemorrhages before 1980. Failure of bleeding control with SB tube was an independent risk factor related to overall mortality. TIPS was not performed in all patients with failure of bleeding control, because emergency TIPS is not available in our center. In cases with success of bleeding control, overall mortality rate was only $22.0 \%$. In addition, endotracheal intubation before SB tube was an independent risk factor related to overall mortality.

When treating patients with failed endoscopic therapies, TIPS can be an extremely effective treatment for controlling variceal hemorrhages. Moreover, patients with Child-Pugh class C disease or class B disease with active bleeding showed a significant decrease in bleeding and mortality if they were treated with TIPS early in their diagnosis [24]. However, incidences of encephalopathy and mortality remain high in patients with poor liver function (Child B or C) [25]. In addition, TIPS insertion is a technically challenging procedure and is not available at all centers. Recently, studies have introduced an interesting alternative to balloon tamponade, the esophageal stent, which has also been proposed as a way to bridge the gap of intervention therapy [26-28]. In small, noncontrolled pilot studies the self-expanding stents were found to control for initial refractory bleeding in $70 \%$ to $100 \%$ of patients [26-28]. However, stent migration to the stomach was observed in several cases. Further studies, including a larger number of patients, are necessary to confirm these data.

To our knowledge, this was the first study to show clinical outcomes of patients treated with SB tube for uncontrolled variceal hemorrhage, since the era of the endoscopic band ligation. However, our study had some limitations. First, the study was conducted in a retrospective cohort. Second, we excluded patients in whom endoscopy could not be performed including patients with severe variceal bleeding who had died before endoscopy due to excessive bleeding.

In conclusion, SB tube might be a beneficial option in rescue therapy for uncontrolled variceal hemorrhage if emergency TIPS is not available, but lethal esophageal complications due to SB tube still occurred. Lower Child-Pugh score and non-intubated state before SB tube insertion were independent factors for initial hemostasis with SB tube. In addition, failure of initial hemostasis with SB tube and endotracheal intubation before SB tube were independent risk factors related to overall mortality. Based on these findings, we recommend careful insertion of the SB tube, especially in patients with endotracheal intubation. The role of SB tube for rescue therapy in patients with uncontrolled variceal bleeding warrants further study.

\section{KEY MESSAGE}

1. Since the era of endoscopic band ligation, Sengstaken-Blakemore (SB) tube might be a beneficial option in rescue therapy for uncontrolled variceal hemorrhage associated with hemody- 
namic instability or failure of endoscopic treatment.

2. However, SB tube had a high incidence of fatal complication and rebleeding.

\section{Conflict of interest}

No potential conflict of interest relevant to this article was reported.

\section{Acknowledgements}

We are grateful for the dedication of our collaborators and research coordinators (Jeong Woo Hong and Hyun Seon You).

\section{REFERRENCES}

1. de Franchis R; Baveno V Faculty. Revising consensus in portal hypertension: report of the Baveno $\mathrm{V}$ consensus workshop on methodology of diagnosis and therapy in portal hypertension. J Hepatol 2010;53:762-768.

2. Chalasani N, Kahi C, Francois F, et al. Improved patient survival after acute variceal bleeding: a multicenter, cohort study. Am J Gastroenterol 2003;98:653-659.

3. Rajoriya N, Tripathi D. Historical overview and review of current day treatment in the management of acute variceal haemorrhage. World J Gastroenterol 2014;20:64816494 .

4. Carbonell N, Pauwels A, Serfaty L, Fourdan O, Levy VG, Poupon R. Improved survival after variceal bleeding in patients with cirrhosis over the past two decades. Hepatology 2004;40:652-659.

5. Colapinto RF, Stronell RD, Gildiner M, et al. Formation of intrahepatic portosystemic shunts using a balloon dilatation catheter: preliminary clinical experience. AJR Am J Roentgenol 1983;140:709-714.

6. Sengstaken RW, Blakemore AH. Balloon tamponage for the control of hemorrhage from esophageal varices. Ann Surg 1950;131:781-789.

7. Conn HO, Simpson JA. Excessive mortality associated with balloon tamponade of bleeding varices: a critical reappraisal. JAMA 1967;202:587-591.

8. Hunt PS, Korman MG, Hansky J, Parkin WG. An 8-year prospective experience with balloon tamponade in emer- gency control of bleeding esophageal varices. Dig Dis Sci 1982;27:413-416.

9. Feneyrou B, Hanana J, Daures JP, Prioton JB. Initial control of bleeding from esophageal varices with the Sengstaken-Blakemore tube: experience in 82 patients. Am J Surg 1988;155:509-511.

10. Panes J, Teres J, Bosch J, Rodes J. Efficacy of balloon tamponade in treatment of bleeding gastric and esophageal varices: results in 151 consecutive episodes. Dig Dis Sci 1988;33:454-459.

11. Sarin SK, Nundy S. Balloon tamponade in the management of bleeding oesophageal varices. Ann R Coll Surg Engl 1984;66:30-32.

12. Bosch J, Abraldes JG, Berzigotti A, Garcia-Pagan JC. Portal hypertension and gastrointestinal bleeding. Semin Liver Dis 2008;28:3-25.

13. Moore KP, Wong F, Gines P, et al. The management of ascites in cirrhosis: report on the consensus conference of the International Ascites Club. Hepatology 2003;38:258266.

14. Chojkier M, Conn HO. Esophageal tamponade in the treatment of bleeding varices: a decadel progress report. Dig Dis Sci 1980;25:267-272.

15. Nielsen TS, Charles AV. Lethal esophageal rupture following treatment with Sengstaken-Blakemore tube in management of variceal bleeding: a 10-year autopsy study. Forensic Sci Int 2012;222:e19-e22.

16. Sorbi D, Gostout CJ, Peura D, et al. An assessment of the management of acute bleeding varices: a multicenter prospective member-based study. Am J Gastroenterol 2003;98:2424-2434.

17. Avgerinos A, Armonis A. Balloon tamponade technique and efficacy in variceal haemorrhage. Scand J Gastroenterol Suppl 1994;207:11-16.

18. Bauer JJ, Kreel I, Kark AE. The use of the Sengstaken-Blakemore tube for immediate control of bleeding esophageal varices. Ann Surg 1974;179:273-277.

19. Chong CF. Esophageal rupture due to Sengstaken-Blakemore tube misplacement. World J Gastroenterol 2005;11:6563-6565.

20. Pinto Correia J, Martins Alves M, Alexandrino P, Silveira J. Controlled trial of vasopressin and balloon tamponade in bleeding esophageal varices. Hepatology 1984;4:885-888.

21. Teres J, Planas R, Panes J, et al. Vasopressin/nitroglycerin infusion vs. esophageal tamponade in the treatment of acute variceal bleeding: a randomized controlled trial. 
Hepatology 1990;11:964-968.

22. Jaramillo JL, de la Mata M, Mino G, Costan G, Gomez-Camacho F. Somatostatin versus Sengstaken balloon tamponade for primary haemostasia of bleeding esophageal varices: a randomized pilot study. J Hepatol 1991;12:100-105.

23. McKee R. A study of octreotide in oesophageal varices. Digestion 1990;45 Suppl 1:60-64.

24. Garcia-Pagan JC, Caca K, Bureau C, et al. Early use of TIPS in patients with cirrhosis and variceal bleeding. $\mathrm{N}$ Engl J Med 2010;362:2370-2379.

25. Riggio O, Angeloni S, Salvatori FM, et al. Incidence, natural history, and risk factors of hepatic encephalopathy after transjugular intrahepatic portosystemic shunt with polytetrafluoroethylene-covered stent grafts. Am J Gastroenterol 2008;103:2738-2746.

26. Hubmann R, Bodlaj G, Czompo M, et al. The use of self-expanding metal stents to treat acute esophageal variceal bleeding. Endoscopy 2006;38:896-901.

27. Zehetner J, Shamiyeh A, Wayand W, Hubmann R. Results of a new method to stop acute bleeding from esophageal varices: implantation of a self-expanding stent. Surg Endosc 2008;22:2149-2152.

28. Wright G, Lewis H, Hogan B, Burroughs A, Patch D, O'Beirne J. A self-expanding metal stent for complicated variceal hemorrhage: experience at a single center. Gastrointest Endosc 2010;71:71-78. 